\title{
EVALUACIÓN DE FACTORES ABIÓTICOS QUE INCIDEN SOBRE LA GERMINACIÓN DE DOS BIOTIPOS DE ARROZ ROJO (Oryza sativa L.)
}

\section{Enrique Quevedo García1 ., Martha Esperanza Arévalo González² ., Giovanni Orlando Cancino Escalante ${ }^{3}$}

\footnotetext{
1 Universidad de Pamplona. Profesor Asociado, Departamento de Agronomía.

2 Universidad Nacional de Colombia.

3 Universidad de Pamplona.Departamento de Biología.Facultad de Ciencias Básicas
}

\section{Resumen.}

El arroz rojo es una maleza importante en muchos campos de arroz. El objetivo de este estudio fue evaluar los efectos de la luz, reguladores del crecimiento y KNO3 sobre el porcentaje de germinación (\%G) y velocidad de la misma (ITG) en dos biotipos de arroz rojo. Se utilizó un diseño completamente al azar con arreglo factorial con cuatro repeticiones de 50 semillas, dos biotipos, ocho factores y 22 niveles. La técnica usada posterior al análisis de varianza fue la de Bonferroni. Los biotipos pipón desgranador y mechudo patechulo se comportaron en forma diferente respecto al ITG y \%GER para cada uno de los tratamientos aplicados. La germinación fue inhibida por las concentraciones de FAP $10^{-2} \mathrm{M}$. El proceso de germinación se vio favorecido para los biotipos por

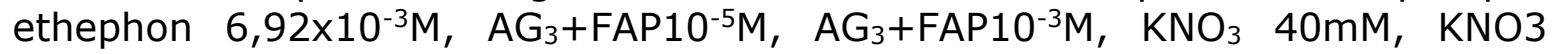
$30 \mathrm{mM}, \mathrm{KNO}_{3} 20 \mathrm{mM}, 20 / 30^{\circ} \mathrm{C}$, Luz amarilla y PEG $\left(30^{\circ} \mathrm{C}\right)$. La ITG se vio favorecida para los biotipos por ethephon $6,92 \times 10^{-3} \mathrm{M}$, FAP $10^{-5} \mathrm{~m}, \mathrm{KNO}_{3} 40 \mathrm{Mm}$, $\mathrm{KNO}_{3} 30 \mathrm{mM}, \mathrm{KNO}_{3} 20 \mathrm{mM}, 20^{\circ} \mathrm{C}, 20 / 30^{\circ} \mathrm{C}$, y PEG. El tratamiento ethephon $6,92 \times 10^{-3} \mathrm{M}, \mathrm{KNO}_{3}$ en sus concentraciones 40,30 y $20 \mathrm{mM}$, la alternancia de temperaturas de $20 / 30^{\circ} \mathrm{C}$ y $30^{\circ} \mathrm{C}$ (PEG) rompen de mejor manera la dormancia de los biotipos. Los tratamientos en común para inducir la germinación y el ITG de los biotipos deben ser probados en bioensayos en otros grupos de arroz rojo como los rayones y varietales que también presentan dormancia.

Palabras clave: Arroz rojo, Arroz maleza, biotipos,dormancia, germinación, velocidad de germinación.

\section{EVALUATION OF FACTORS AFFECTING THE ABIOTIC GERMINATE BIOTYPES TWO RED RICE (O. sativa L.)}

\section{ABSTRACT}

Red rice is considered a weed in many rice fields. The aim of this study was to evaluate the effects of light, growth regulators and $\mathrm{KNO}_{3}$ on the germination 
64

percentage (G\%) and germination rate index (GRI) in two red rice biotypes. A random design, with factorial arrangement, four repetitions of 50 seeds, 2 biotypes, 8 factors and 20 levels was used. The technique used subsequent to the analysis of variance was Bonferroni's multiple-comparisons test. Biotypes of red "pipón threshing" rice and "mechudo patechulo" behaved differently with respect to the GRI and G\% for each of the treatments applied. Germination was inhibited by the effect of the concentrations of FAP $10-2 \mathrm{M}$. The germination process for the two biotypes was benefitted by ethephon 6,92×10-3M, GA 3 +FAP 10-5 M, GA3+FAP 10-3M, $\mathrm{KNO}_{3} 40 \mathrm{mM}, \mathrm{KNO} 330 \mathrm{mM}, \mathrm{KNO} 320 \mathrm{mM}, 20 / 30^{\circ} \mathrm{C}$, yellow light and PEG $\left(30^{\circ} \mathrm{C}\right)$. The GRI was favoured for the two biotypes by ethephon $6.92 \times 10^{-3} \mathrm{M}, \mathrm{FAP} 10^{-5} \mathrm{~m}, \mathrm{KNO}_{3} 40 \mathrm{Mm}, \mathrm{KNO}_{3} 30 \mathrm{mM}, \mathrm{KNO}_{3} 20 \mathrm{mM}, 20^{\circ} \mathrm{C}$, $20 / 30^{\circ} \mathrm{C}$ and SGT. The ethephon treatment of $6.92 \times 10^{-3} \mathrm{M}, \mathrm{KNO}_{3}$ in its different concentration $\left(40,30\right.$ and $20 \mathrm{~mm}$ ) and the changes in temperature $20 / 30^{\circ} \mathrm{C}$ and $30^{\circ} \mathrm{C}$ (SGT) were the best alternative in breaking the biotypes dormancy. Common treatments to induce germination and the GRI of biotypes must be tested in bioassays in other groups of red rice that also present dormancy.

Keyword: Rice rice, Weedy Rice, Dormancy, Germination, Genotypes, Germination speed.

Para citar este artículo: Quevedo García E., Arévalo González ME ., Cancino Escalante GO.Evaluación de factores abióticos que inciden sobre la germinación de dos biotipos de arroz rojo (Oryza sativa L).. Revista Bistua.2015.13(2):63-78

+ Autor para el envió de correspondencia y la solicitud de las separatas: Quevedo García E. Departamento de Agronomía. Universidad de Pamplona. Norte de Santander- Colombia. email: enriquegarcia@unipamplona.edu.co

\section{Introducción}

El AR (Arroz rojo) es común en Argentina, Bolivia, Brasil, Chile, China, Colombia, Costa rica, Ecuador, Guyana, Estados Unidos, España, Filipinas, Grecia, Honduras, India, Malasia, México, Nicaragua, Panamá, Perú, Portugal, República Dominicana, Sri Lanka, Uruguay, Venezuela, Vietnam y Tailandia ${ }^{1-3}$.
Las infestaciones de AR en las zonas arroceras van en aumento, lo que causa pérdidas en rendimientos y calidad $^{4}$. Las pérdidas de arroz paddy debidas a la interferencia de malezas que escapan a las prácticas de control son del $10 \%$ de la producción agronómica mundial, estimada en 46 millones de ton de grano/año $0^{5}$ con AR llega al $20 \%{ }^{6}$, 
debido a que es similar taxonómica y fisiológicamente al cultivo, es la principal maleza asociada al cultivo de arroz y se disemina, por el uso de semillas contaminadas, equipos agrícolas y la siembra directa de los cariópsides de arroz ${ }^{2}$.

La diversidad genética de los biotipos de $A R$ es alta 2,5,6. Evolutivamente los AR tienden a divergir del arroz cultivado en las tasas fotosintéticas que se presentan por latitud y temperatura ${ }^{7}$. Los AR japónicos realizan la máxima fotosíntesis entre $18-35{ }^{\circ} \mathrm{C}$ y los materiales de origen índico entre 25$35{ }^{\circ} \mathrm{C}$ lo que demuestra una respuesta diferencial a cambios ambientales ${ }^{8}$. En condiciones naturales, para germinar los AR tienen que someterse a la alternancia de dos temperaturas, este tratamiento se denomina termoperiodicidad. Las temperaturas cardinales de germinación se ubican en los siguientes rangos: mínima 10 a $12^{\circ} \mathrm{C}$, óptima de 30 a $37^{\circ} \mathrm{C}$ y máxima de 40 a $42^{\circ} \mathrm{C}^{9}$. La temperatura crítica de germinación se correlaciona con la temperatura local y el gradiente latitudinal del hábitat del $A R^{7}$, indicando que la germinación responde en parte a cambios climáticos ${ }^{10}$.La dormancia de las semillas de PD y MP está regida por la permeabilidad de las estructuras al paso del oxígeno o de cualquier otro iniciador de rutas metabólicas 0 inhibidor de ellas ${ }^{11}$. La presencia de la dormancia es la principal característica fisiológica que dificulta el control de AR, ya que distribuye la germinación a lo largo del tiempo ${ }^{12}$ y ha evolucionado como mecanismo de sobrevivencia, cuando las etapas vegetativas y reproductivas del ciclo anual se han
65

completado.EI AR proveniente de lotes de semilla certificada se parecen a las variedades de arroz y están evolucionando a AR varietales para mimetizarse con los cultivares de arroz, este grupo de AR presenta mayor dormancia en sus semillas dificultando el control agronómico. Los biotipos de AR más frecuentes en los distritos de riego de Colombia como el del Zulia en Norte de Santander son Mechudo patechulo (MP) y pipón desgranador (PD). En "pipones" el grano puede ser corto, intermedio o largo, ancho y tiene un ligero abultamiento en la parte media, por esto se denominan pipones, sus glumas son de color pajizo y su porcentaje de desgrane es superior al de los otros grupos de AR (Varietales, rayones y mechudos), presenta larga dormancia de las semillas (de 55 a 70 días) y tienen buena estabilidad genética. Los "Mechudos" se caracterizan por arista larga en el grano y aplanada. Las glumas del grano son de colores diferentes que varían entre el negro al marrón y el pajizo ${ }^{11}$.El presente bioensayo se desarrolló con el fin de evaluar los efectos sobre el porcentaje de germinación y velocidad de la misma (ITG) de dos biotipos de $A R$, que puedan tener la luz, temperatura, algunos reguladores de crecimiento y el nitrato de potasio.

\section{MÉTODOS}

Se recolectaron cariópsides maduras de pipón desgranador (PD) y mechudo patechulo (MP) de la zona arrocera del Zulia Norte de Santander Colombia. Tras la recolección, el lote de cariópsides fue guardado durante seis meses en un 
recipiente plástico de color amarillo que no dejaba entrar la luz y ambiente seco en el laboratorio de biotecnología de la Universidad de Pamplona. Posteriormente fueron tratadas con tiabendazol (dosis de $0,91000 \mathrm{cc}^{-1}$ de agua destilada) y el laboratorio con un limpiador higienizante concentrado, con alto poder desengrasante y antibacterial. Se usó el hipoclorito de sodio al 5\% para desinfectar los materiales antes de la siembra. Los cariópsides se colocaron en 88 placas plásticas de Petri con un diámetro de $12 \mathrm{~cm}$ debidamente marcadas sobre papel de filtro Whatman No. 3 humedecido con $10 \mathrm{~mL}$ de agua destilada y esterilizada $(\mathrm{pH} \mathrm{5,5)}$ o de solución con una pipeta de $10 \mathrm{~mL}$ a la concentración de la sustancia que se reponía cuyo era necesario (unas dos veces a la semana) según la tabla 1 , para prepararlas se utilizó una balanza analítica, 14 Erlenmeyer, 2 probetas $(100$ y $1000 \mathrm{~mL}), 1$ embudo de vidrio, 1 espátula y un vaso de precipitados de $500 \mathrm{~mL}$.Las placas se colocaron a $30^{\circ} \mathrm{C}$ en una cámara climática de germinación Seedburo Equipment Company donde se mantuvo la temperatura constante y la humedad relativa cercana al punto de saturación a fin de evitar la desecación de los sustratos, permitió trabajar a temperaturas que diferían de la temperatura ambiente y estaba prevista de luz, con capacidad de 10 bandejas. Se usó para las pruebas de germinación la prueba estándar de germinación (PEG) según protocolo establecido por el ISTA $^{13}$ ,manteniendo una temperatura de $30^{\circ} \mathrm{C}$ durante el periodo de oscuridad de 12 horas y el periodo de luz de 12 horas. Para cada tratamiento se utilizaron cuatro placas de Petri que contenían 50 cariópsides embebidos en soluciones respectivas. Los datos de germinación se registraron a los 4,8 y 12 días, se retiró en cada observación cariópsides germinadas.

El criterio de germinación fue la protrusión visible del coleóptilo. El experimento se mantuvo hasta que no se obtuvieron más germinaciones tras 12 días consecutivos. La germinación se expresó en porcentaje (\%G) y como velocidad de germinación se expresó como el índice de la tasa de germinación (ITG), que fue calculado como:

ITG $=\left(G_{1} / T_{1}+G_{2} / T_{2}+\ldots+G_{n} / T_{n}\right) / \% G$, donde:

$\mathrm{G}_{1}=$ porcentaje de cariópsides germinadas en $T_{1}, G_{2}=$ porcentaje de cariópsides entre $T_{1}$ y $T_{2} . G_{n}=$ porcentaje de cariópsides germinadas al final, $\mathrm{T}_{1}=$ días al primer conteo, $\mathrm{T}_{2}=$ días al segundo conteo, $T_{n}=$ días al conteo final, $\% G$ $=$ porcentaje total de germinación.

Prueba estándar de germinación (PEG)

La prueba se hizo con una temperatura de $30^{\circ} \mathrm{C}$ en forma constante, 12 horas de luz y 12 horas de oscuridad (PEG).

\section{Luz}

Los espectros azul (390-590nm) y rojo (580-680nm) se lograron envolviendo las cajas con papel celofán. En el caso del rojo lejano, éste fue obtenido mediante dobles envolturas en celofán azul y del rojo. Las características ópticas del celofán fueron determinadas en un fotómetro Spectronic $20^{14}$. Los datos se registraron en un cuarto oscuro bajo luz de seguridad verde durante 
12 días, cada cuatro días. Los niveles aplicados se muestran en la tabla 1.

\section{Temperatura}

Para determinar el efecto de temperaturas altas y bajas sobre el porcentaje de germinación e ITG, se usaron los niveles $20^{\circ} \mathrm{C}, 40^{\circ} \mathrm{C}$ y 20/30 ${ }^{\circ} \mathrm{C}$ simultáneamente en la cámara de germinación. Las lecturas se realizaron durante 12 días.

\section{Sustancias metabólicas y reguladores del crecimiento}

Las cariópsides se sometieron a imbibición en diferentes sustancias metabólicas y reguladores de crecimiento, para evaluar el efecto de los mismos. Las soluciones se prepararon en las concentraciones que se muestran en la tabla 1 . Se usó alcohol etílico al $96 \%$ para diluir las sustancias hormonales Ácido giberélico $\left(\mathrm{AG}_{3}\right)$ y 6 -furfuril amino purina (FAP). Para calcular la concentración de las soluciones se tuvo en cuenta los siguientes pesos moleculares de las sustancias citoquinina en forma de FAP $(215 \mathrm{~g} / \mathrm{mol})$, giberelina en forma de $\mathrm{AG}_{3}(346,38 \mathrm{~g} / \mathrm{mol}), \mathrm{KNO}_{3}(101,10$ $\mathrm{g} / \mathrm{mol}$ ) y etileno en forma de ethephon $(144,5 \mathrm{~g} / \mathrm{mol})$.

\section{Estadísticas}

Para analizar los datos se usó un diseño experimental completamente al azar con arreglo factorial de dos biotipos, ocho factores y 22 niveles, los datos fueron procesados con el paquete SAS 9.1 (Statistical Analysis System). Las técnicas usadas posteriores al análisis de varianza (anova) fue la de comparación múltiple de medias de Bonferroni.

\section{RESULTADOS}

El análisis de varianza mostro que el modelo factorial fue significativo a $P$ $\leq 0,001$ para biotipos, factores $y$ niveles. Además la interacción entre
67

biotipos, factores $y$ niveles fue significativa a $P \leq 0,001$ para la variable germinación pero no fue significativa en la interacción entre las tres fuentes de variación para ITG como se puede ver en la tabla 2 . Los $\mathrm{R}^{2}$ de los modelos estuvieron por encima del $50 \%$ y los coeficientes de variación por debajo del 30 \% para las variables respuesta. Los resultados y análisis se centraron en las interacciones de los tres factores usando la prueba de Bonferroni.

\section{Interacción Biotipo Factor $y$ Nivel.Etileno}

Los mejores resultados obtenidos fue para PD fue con ethephon $6,92 \times 10^{-3}$ $M$ y de $91 \%$ y y se diferenció a un $\mathrm{p}<0,05$ de $\mathrm{LA}, 40^{\circ} \mathrm{C}, \mathrm{AG}_{3} 10^{-3} \mathrm{M}, \mathrm{AG}_{3}$ $10^{-5} \mathrm{M}, 20^{\circ} \mathrm{C}$, FAP $10^{-5} \mathrm{M}$, FAP $10^{-3} \mathrm{M}$, $\mathrm{AG}_{3}+\mathrm{FAP} \quad 10^{-3} \mathrm{M} \quad 5 \mathrm{~h}$, ethephon $1,4948 \times 10^{-2} \mathrm{M}$ y FAP $10^{-2} \mathrm{M}$. El mejor tratamiento ethephon $6,92 \times 10^{-3} \mathrm{M}$ formo subgrupo con los tratamientos LAM, $\mathrm{AG}_{3}+\mathrm{FAP} 10^{-3} \mathrm{M}$ 3h, $\mathrm{AG}_{3}+\mathrm{FAP}$ $10^{-5} \mathrm{M}, \mathrm{KNO}_{3} 20 \mathrm{Mm}$, luz roja/azul, $\mathrm{KNO}_{3} 30 \mathrm{mM}, \mathrm{AG}_{3}+\mathrm{FAP} 10^{-3} \mathrm{M}, \mathrm{LR}$, $20 / 30^{\circ} \mathrm{C}, \mathrm{KNO}_{3} 40 \mathrm{mM}$ y PEG como se muestra en la tabla 3. Para PD en el ITG el mejor tratamiento fue el mismo ethephon $6,92 \times 10^{-2} \mathrm{M}$ con un valor de 0,2325 y fue diferente a un $\mathrm{p}<0,05$ de $\mathrm{AG}_{3}+\mathrm{FAP} 10^{-3} \mathrm{M}$, ethephon $1,4948 \times 10^{-2} \mathrm{M}, \quad \mathrm{AG}_{3} \quad 10^{-3} \mathrm{M}$, $\mathrm{AG}_{3}+\mathrm{FAP} 10^{-3} \mathrm{M}$ 3h., $\mathrm{AG}_{3} 10^{-5} \mathrm{M}$, FAP $10^{-3} \mathrm{M}$ y de FAP $10^{-2} \mathrm{M}$ que fue el peor tratamiento y tuvo un valor de 0,1550 . El mejor tratamiento para PD formo un subgrupo con PEG, $\mathrm{KNO}_{3} 30 \mathrm{mM}, 20^{\circ} \mathrm{C}, \mathrm{AG}_{3}+\mathrm{FAP} 10^{-5} \mathrm{M}$, FAP $10^{-5} \mathrm{M}, 20 / 30^{\circ} \mathrm{C}, \mathrm{KNO}_{3} 20 \mathrm{mM}$, $\mathrm{LR}, \mathrm{KNO}_{3} 40 \mathrm{mM}, 40^{\circ} \mathrm{C}$, luz roja/azul, LAM, LA y $\mathrm{AG}_{3}+\mathrm{FAP} 10^{-3} \mathrm{M} 5 \mathrm{~h}$, como se muestra en la tabla 3. El ethephon $6,92 \times 10^{-3} \mathrm{M}$ en el ecotipo MP formo el mejor subgrupo con $\mathrm{KNO}_{3} 20 \mathrm{mM}$, $\mathrm{AG}_{3}$ +FAP $10^{-5} \mathrm{M}, \mathrm{AG}_{3}+\mathrm{FAP} 10^{-3} \mathrm{M}$, 
$\mathrm{KNO}_{3} 30 \mathrm{mM}, \mathrm{PEG}, \mathrm{AG}_{3} 10^{-3} \mathrm{M}, \mathrm{KNO}_{3}$ $20 \mathrm{mM}, \mathrm{AG}_{3} 10^{-5} \mathrm{M}, 20 / 30^{\circ} \mathrm{C}$, $\mathrm{AG}_{3}+\mathrm{FAP} 10^{-3} \mathrm{M} 5 \mathrm{~h}, \mathrm{LAM}, 20^{\circ} \mathrm{C}$ y LA como se muestra en la tabla 3 . El mejor subgrupo para la variable respuesta ITG en MP fue el conformado por $\mathrm{AG}_{3} 10^{-3} \mathrm{M}$, ethephon $6,92 \times 10^{-3} \mathrm{M}, \mathrm{KNO}_{3} 20 \mathrm{mM}, \mathrm{KNO}_{3} 30$ $\mathrm{mM}, \mathrm{PEG}, \mathrm{FAP} 10^{-5} \mathrm{M}, 20^{\circ} \mathrm{C}, \mathrm{AG}_{3}+\mathrm{FAP}$ $10^{-3} \mathrm{M} 5 \mathrm{~h}, \mathrm{KNO}_{3} 40 \mathrm{mM}, \mathrm{AG}_{3} 10^{-5} \mathrm{M}$, 20/30 ${ }^{\circ} \mathrm{C} \mathrm{AG}_{3}+\mathrm{FAP} 10^{-3} \mathrm{M}$ 3h y Luz roja/azul. El mejor subgrupo que promovió la germinación fue diferente a $p<0,05$ de los tratamientos $40^{\circ} \mathrm{C}$, LR, FAP $10^{-3} \mathrm{M}$, FAP $10^{-5} \mathrm{M}$, ethephon $1,4948 \times 10^{-2} \mathrm{M}$, luz roja/azul y de FAP $10^{-2} \mathrm{M}$ que fue el peor tratamiento con $5 \% \mathrm{G}$.

\section{Giberelinas y Citoquininas}

Para ITG en MP el máximo valor se alcanzó con el tratamiento $\mathrm{AG}_{3} 10^{-3} \mathrm{M}$ con un valor de 0,2325 y fue diferente a un $p<0,05$ de los tratamientos $\mathrm{AG}_{3}+\mathrm{FAP} 10^{-3} \mathrm{M}, 40^{\circ} \mathrm{C}$, FAP $10^{-3} \mathrm{M}, \mathrm{AG}_{3}+\mathrm{FAP} 10^{-5} \mathrm{M}$, LR, LAM, $L A$, ethephon $1,4948 \times 10^{-2} \mathrm{M}$ y FAP $10^{-2} \mathrm{M}$ que fue el peor tratamiento de todos y se diferenció de los demás a un nivel de $\mathrm{P}<0,05$ como se muestra en la tabla 3. Para MP en el grupo con el mejor tratamiento ethephon 6,92 $\times 10^{-3} \mathrm{M}$ que tuvo $91 \% \mathrm{G}$. En cuanto a las citoquininas (FAP) el mejor tratamiento fue FAP $10^{-5} \mathrm{M}$ con $43,50 \%$, luego FAP $10^{-3} \mathrm{M}$ con $41,00 \%$ y el peor nivel fue FAP $10^{-}$ $2 M$ con $15,50 \%$ el cual inhibió la germinación de los biotipos como se muestra en la tabla 3.

\section{Nitratos}

Los resultados que se muestran en la tabla 3, indican que las tres concentraciones de $\mathrm{KNO}_{3}$ se ubicaron entre los mejores subgrupos para estimular la velocidad y el porcentaje de germinación de los dos biotipos de 68
$A R$, el rango de concentración de los tratamientos se ubicó entre los 20 a 40 micromoles. El mejor tratamiento para estimular la germinación en PD fue KNO3 $40 \mathrm{mM}$ con $68,00 \%$ y para ITG fue $\mathrm{KNO}_{3}$ con $30 \mathrm{mM}$. En el caso del biotipo MP el mejor tratamiento para estimular la germinación fue $\mathrm{KNO}_{3} 40 \mathrm{mM}$ con $71,50 \%$ y para ITG fue $\mathrm{KNO}_{3} 20 \mathrm{mM}$ con 0,2175.

\section{Temperatura}

El tratamiento PEG (Con $30^{\circ} \mathrm{C}$ ) alcanzo para PD un valor de $57,06 \% \mathrm{G}$ y se diferenció a un $\mathrm{p}<0,05$ de FAP $10^{-2} \mathrm{M}$ y etephon $1,4948 \times 10^{-}$ ${ }^{2} \mathrm{M}$ y formo 3 subgrupos con los otros tratamientos y para ITG se obtuvo con este tratamiento 0,2306 y se diferenció a un nivel de $p<0,05$ de FAP $10^{-3} \mathrm{M}$ y FAP $10^{-2} \mathrm{M}$ como muestra la tabla 3, para MP, PEG alcanzo un valor de $53,94 \% \mathrm{G}$ y se diferenció a un $p<0,05$ del tratamiento FAP $10^{-2} \mathrm{M}$ y para ITG se obtuvo un máximo valor de 0,2028 y se diferenció a un nivel de $\mathrm{p}<0,05$ de etephon $1,4948 \times 10^{-2} \mathrm{M}$ y FAP $10^{-2} \mathrm{M}$ como se muestra en la tabla 3.

Para el PD se obtuvo un valor de $60 \% \mathrm{G}$ con el tratamiento $20 / 30^{\circ} \mathrm{C}$, $57,06 \%$ a $30^{\circ} \mathrm{C}$ (PEG), y estos se diferenciaron a un $\mathrm{p}<0,05$ de los otros tratamientos con temperatura que fueron que dieron como resultado $53,50 \% \mathrm{G}$ para $40^{\circ} \mathrm{C}$ y $49,0 \% \mathrm{G}$ para $20^{\circ} \mathrm{C}$, y además presentaron diferencias a un $p<0,05$ con $\mathrm{FAP} 10^{-2} \mathrm{M}$ que tuvo un $15,50 \%$. Para el caso del ITG se obtuvo un máximo valor con el tratamiento con $30^{\circ} \mathrm{C}$ (PEG) y 0,2306 , luego siguió $20^{\circ} \mathrm{C}$ con $0,2150,20 / 30^{\circ} \mathrm{C}$ con 0,2075 y por ultimo $40^{\circ} \mathrm{C}$ con 0,2025 . Los tratamientos $30^{\circ} \mathrm{C}, 20 / 30^{\circ} \mathrm{C}$ y $20^{\circ} \mathrm{C}$ fueron diferentes a un $p<0,05$ de FAP $10^{-2} \mathrm{M}$ que fue el peor tratamiento 
para incrementar ITG tal como se muestra en la tabla 3.

Para MP se obtuvo a $30^{\circ} \mathrm{C}$ (PEG) el mayor valor con $53,94 \% G$ y se diferenció a un $\mathrm{p}<0,05$ de FAP $10^{-2} \mathrm{M}$, luego lo siguió $20 / 30^{\circ} \mathrm{C}$ con $48,50 \% \mathrm{G}, 20^{\circ} \mathrm{C}$ con $42 \% \mathrm{G}$, y $40^{\circ} \mathrm{C}$ con $36,50 \%$, el ultimo tratamiento se diferenció a un nivel de $\mathrm{p}<0,05$ de los mejores tratamientos ethephon 6,92x10-3 $\mathrm{M}$ y $\mathrm{KNO}_{3} 40 \mathrm{mM}$. El tratamiento $40^{\circ} \mathrm{C}$ para $\mathrm{MP}$ formo un subgrupo con los peores tratamientos para inducir la germinación $\mathrm{AG}_{3}+\mathrm{FAP} 10^{-3} \mathrm{M} 3 \mathrm{~h}$, LR, FAP $10^{-2} \mathrm{M}$, FAP $10^{-3} \mathrm{M}$, ethephon $1,4948 \times 10^{-2} \mathrm{M}$, luz roja/azul y FAP $10^{-5} \mathrm{M}$.

\section{Luz}

Para el biotipo PD el tratamiento de mejor comportamiento fue LAM y formo un subgrupo con luz roja/azul y LR. Por otro lado un subgrupo de tratamientos se formó con LA, que a su vez se diferenció a un $p<0,05$ de los peores tratamientos para promover la germinación como fueron $\mathrm{AG}_{3}+\mathrm{FAP} 10^{-5} 5 \mathrm{~h}$, etephon $1,4948 \times 10^{-2} \mathrm{M}$ y FAP $10^{-2} \mathrm{M}$ y en el caso del ITG el mejor tratamiento fue LR con un valor de 0,2025, luego roja/azul, LAM y por último la LA con 0,19 , como se muestra en la tabla 3. Para MP el mejor tratamiento fue LAM con un valor de $42,50 \% G$ y se diferenció a un nivel de $p<0,05$ de FAP $10^{-2} \mathrm{M}$, luego siguió LA, posteriormente LR y por último la azul/roja. La LA presentó diferencias a un $\mathrm{p}<0,05$ de FAP $10^{-2} \mathrm{M}$. Para el caso del ITG el mejor tratamiento luz roja/azul con 0,1775 , luego LR con 0,16 , la LAM con 0,16 y por último la LA con 0,1575 , los tratamientos de luz para MP hicieron subgrupo con el peor tratamiento que fue FAP $10^{-2} \mathrm{M}$ tal como se muestra en la tabla 3.
DISCUSIÓN

Interacción Biotipo Factor y Nivel.Etileno

Para MP y PD el mejor tratamiento fue ethephon $6,29 \times 10^{-3} \mathrm{M}$ con $73 \% \mathrm{G}$ que promovió la $\% \mathrm{G}$, lo mismo ocurrió con los tratamientos que la aplicación exógena de los tratamientos con $\mathrm{KNO}_{3}, \mathrm{AG}_{3}$ y la combinación de $\mathrm{AG}_{3}$ y $\mathrm{BAP}^{15-17}$. La aplicación de etileno en forma de ethephon $\left(6,9 \times 10^{-3} \mathrm{M}\right)$ en bajas concentraciones trajo como consecuencia fisiológica modificaciones precoces en la permeabilidad de las membranas de las células de los cariópsides de los biotipos, evento que ayudo a mejorar la germinación ${ }^{15}$ 18, 19 .

\section{Giberelinas y Citoquininas}

La giberilina es una fitohormona que aplicada exógenamente a concentraciones de $10^{-3}$ es promotora de la germinación de MP al romper su dormancia 15,16, 20-25. El contenido en el cariópside de $\mathrm{AG}_{3}$ se incrementa justo antes de la emergencia de la radícula, lo que sugiere tiene un papel fundamental en la regulación de la germinación, la principal enzima responsable del último paso de la síntesis bioquímica de $A_{3}$ en la semilla es la $A G_{3}-$ oxidasa, la subsecuente degradación de esta fitohormona se hace vía el sitio regulado por la enzima $A_{G_{2}-}$ oxidasa ${ }^{26}$. Lo que confirma que $\mathrm{AG}_{3}$ debe ser suministrado exógenamente y con luz directa para que la semillas de PD y MP empiecen el proceso de germinación ${ }^{25,27}$. La concentración de citoquininas y el tipo de ellas que se use depende la germinación de los biotipos ${ }^{22,28}$, la acción de la citoquinina se inició bajo una cascada de señales que incluye un conjunto de segundos 
mensajeros identificados, reconocidos y cuya función está en la modulación de la respuesta genómica a la acción de la luz roja como se ha hallado en otras especies de $\operatorname{arroz}^{29}$.

La combinación de $\mathrm{AG}_{3}$ +FAP en ciertas concentraciones ayudo a estimular la germinación de PD, porque las citoquininas añadidas exógenamente estimulan la síntesis de ADN y la acción mitógena, es decir la mitosis durante el inicio de la germinación, muestra el efecto conjunto entre FAP y las giberelinas ${ }^{30}$ en la tabla 3. Algunas concentraciones de $\mathrm{AG}_{3}+\mathrm{FAP}$ en el biotipo PD tal como se muestran en la tabla 3 parece que mostraron antagonismo para inducir la germinación ${ }^{30}$.

Por último la germinación de los biotipos se inhibieron con concentraciones de FAP $10^{-2} \mathrm{M}$, evidencio que la aplicación de FAP no funciona cuando se usa en cariópsides con glumas que determina un tipo de dormancia mecánica que impide un mejor control de los AR. Los resultados con las fitohormonas muestran que MP germina más lentamente que PD y por esta característica su control en los campos de cultivo es más difícil.

\section{Nitratos}

El tratamiento $\mathrm{KNO}_{3} 40 \mathrm{mM}$ ayudo al proceso de germinación en los dos biotipos $^{17}, 21$, e indicó que la germinación de los AR es afectada por la concentración de nitratos y el tiempo de exposición del cariópside a la solución de los mismos como fue hallado por $L^{3} i^{31}$. Por otra parte la acción fisiológica de los tratamientos de nitrato en PD y MP se realizó en forma sinergética con la temperatura, la Luz y las giberelinas. 70
Los tratamientos con $\mathrm{KNO}_{3}$ estimularon la elongación de la radícula de los biotipos cuando se usó como fuente los nitratos $8,15,17,21$, 23, y su acción durante la germinación del AR está asociada a la producción de óxido nítrico ${ }^{31}$. La germinación de semillas de los biotipos fue estimulada por nitratos $\left(\mathrm{KNO}_{3}\right)$ en rangos comprendidos entre 0-0,05 mol L-1 15,24. Por último la acción de los nitratos $\left(\mathrm{KNO}_{3}\right)$ sobre el proceso germinativo de los biotipos estuvo asociada con la vía oxidativa de fosfatos de pentosa ${ }^{15,17 .}$

\section{Temperatura}

El uso de temperaturas alternas en el bioensayo ayudaron a incrementar la germinación de PD y $\mathrm{MP}^{17,} 20$ las dos temperaturas alternas cardinales que se utilizaron fueron la óptima $\left(30^{\circ} \mathrm{C}\right)$ y la mínima $\left(20^{\circ} \mathrm{C}\right) . \quad \mathrm{El}$ tratamiento con $30^{\circ} \mathrm{C}$ (PEG) fue el de mejor respuesta para los biotipos en el porcentaje de germinación, e indica la importancia de la temperatura para inducir la germinación teniendo en cuenta los valores cardinales para activar el proceso, además fue afectado en forma deletérea el proceso germinativo por la temperatura de $40^{\circ} \mathrm{C}$. Para el ITG a $30^{\circ} \mathrm{C}$ (PEG) en MP se obtuvo el mayor valor con 0,2028 $y$ este se diferenció a un $p<0,05$ de ethephon $1,4948 \times 10^{-2} \mathrm{M}$ y FAP $10^{-2}$ $M$. Los tratamientos que siguieron en valor fueron $20^{\circ} \mathrm{C}$ con 0,2000 , $20 / 30^{\circ} \mathrm{C}$ con 0,1900 y $40^{\circ} \mathrm{C}$ con 0,1700 . Los tratamientos $20^{\circ} \mathrm{C}$ y $20 / 30^{\circ} \mathrm{C}$ fueron diferentes a un $\mathrm{p}<0,05$ de FAP $10^{-2} \mathrm{M}$ el cual formo subgrupo con el tratamiento $40^{\circ} \mathrm{C}$ y fueron los peores para promover ITG como se muestra en la tabla 3 , e indica que $40^{\circ} \mathrm{C}$ es la temperatura máxima cardinal para que este 
biotipo active sus proceso germinativo.

Como podemos ver para MP la alternancia de temperatura mínima y optima $\left(20 / 30^{\circ} \mathrm{C}\right)$ mejoro la germinación y la ITG de los biotipos como se muestra en la tabla 3 . Es decir, la temperatura influye sobre el proceso de germinación y logro aumentar el \%G e ITG en el PD y MP usando el tratamiento $20 / 30^{\circ} \mathrm{C}$ tal como lo hallado en Corea con materiales asiáticos donde se usó temperaturas de $25^{\circ} \mathrm{C}$ durante 14 horas/ y $20^{\circ} \mathrm{C}$ durante 10 horas ${ }^{6,20 \text {, }}$ 32, 33. El efecto de la temperatura dependió de las condiciones fisiológicas y genéticas de las semillas de los biotipos como podemos ver en las respuestas diferenciales para estos tratamientos en la tabla3. El tratamiento con $20^{\circ} \mathrm{C}$ fue el que menos incremento en la germinación logro sobre los biotipos y nos indica que esta es la temperatura cardinal mínima y que la temperatura óptima para iniciar el proceso fisiológico de germinación y con el cual se obtuvo el mayor valor fue el de $30^{\circ} \mathrm{C}$ usada en el tratamiento PEG para los biotipos.

Por ultimo al parecer las semillas de PD y MP al responder a la alternancia de temperaturas cardinales tales como $20^{\circ} \mathrm{C}$ y $30^{\circ} \mathrm{C}$ se comportan en forma independiente del régimen lumínico y presentan mecanismos enzimáticos que funcionan en diferentes temperaturas y esa respuesta se corresponde probablemente, a una adaptación a las fluctuaciones naturales del ambiente generadas por el manejo agronómico, la alternancia de las temperaturas entre el día y la noche sobre la germinación de los biotipos tal como lo hallaron en otras 71 especies $^{20,32}$, e indicaría que sería otro método alternativo la alternancia para romper la dormancia de estos biotipos debido a la presencia de un complejo mecanismo de tipo físico, primario que está regida por los contenidos de fitohormonas, y secundaria por los factores ambientales como la luz y la temperatura ${ }^{34 .}$

\section{Luz}

La respuesta germinativa observada con respecto al efecto de la luz sobre PD, estuvo asociado con el fitocromo, que controla las respuestas de los cariópsides a la luz evaluando la respuesta de ITG, y que permito establecer que semillas de $\mathrm{PD}$ reaccionaron mejor a $\mathrm{LR}(\mathrm{Luz}$ roja) y a la inducción que las de MP, los resultados que se muestran en la tabla 3 demuestran que para PD, la LR actúa sobre el mecanismo del fitocromo rompiendo la dormancia e induciendo la germinación ${ }^{15,17}$, e indicó que el biotipo MP es más difícil de controlar que el PD en un cultivo de arroz, es decir, los cariópsides de PD presentaron respuesta fotoblástica con mayor intensidad que MP y que la luz fue el estímulo iniciador en combinación con la acción de las fitohormonas como $\mathrm{AG}_{3}+\mathrm{FAP}$ que suplió los requerimientos del sistema de fitocromo en cariópsides de PD e indica que LR es necesaria para inducir la germinación ${ }^{27}$. Los tratamientos de luz estuvieron en el mismo subgrupo con los tratamientos de temperatura con excepción del nivel $40^{\circ} \mathrm{C}$ lo que indica la estrecha relación que existe entre la luz y la temperatura para promover la \%GER de PD y $\mathrm{MP}^{15}$ y demuestra que la LA al ser de los peores tratamientos en los dos 
biotipos promueve la dormancia en gramíneas ${ }^{35}$. Por otra parte la LR en ambos biotipos activa y desactiva el fitocromo que permite a las semillas adaptarse durante la germinación a cambios en las condiciones lumínicas tal como lo hallado para otros materiales genéticos ${ }^{15}$. La presencia de una relación entre le fitocromo rojo/rojo lejano que son reconvertibles establece la razón de la luz roja (FR) con la luz lejana (Pfr) varía notablemente en diferentes medios y se define como la tasa de emisión de fotones en el centro de la banda de $10 \mathrm{~nm}$ sobre $660 \mathrm{~nm} /$ tasa de emisión de fotones en el centro de la banda de $10 \mathrm{~nm}$ sobre $730 \mathrm{~nm}$, representado por los niveles de rojo/rojo lejano que fue de menor importancia en MP y de mayor importancia en PD por ser este último biotipo fotoblástico 15,17,33 .

Como podemos ver en los resultados que están en la tabla 3 con respecto a la luz, están asociados con el fitocromo que regula el metabolismo de ácido abscísico y que esta mediado por la concentración de giberelinas, pigmento que controla las respuestas de las semillas a la luz, con base en los resultados del experimento, se puede inferir que cariópsides de PD presentan la respuesta de un biotipo fotoblástico con respecto a MP y necesitan el estímulo iniciador de sustancias metabólicas como etileno y $\mathrm{GA}_{3}$ sobre la germinación de la misma ${ }^{15,27}$. El tamaño pequeño de PD y MP facilita el proceso de invasión, esta característica permite considerarla como una maleza importante en el sistema de siembra directa, donde los cariópsides germinan desde la superficie del suelo luego de atravesar los residuos 72 vegetales con altos niveles de luz y temperatura en el caso de PD por ser fotoblástica. Lo que indicaría que MP no es fotoblástica y sería una arvense más difícil de controlar teniendo en cuenta que las semillas de variedades de arroz germinan más rápido que las de AR.

\section{CONCLUSIONES}

Los biotipos de arroz rojo pipón desgranador (PD) y mechudo patechulo (MP) se comportaron en forma diferente respecto al \%G e ITG para el efecto de los tratamientos cada uno de los factores y niveles de los mismos.

La germinación se inhibió con concentraciones de FAP $10^{-2} \mathrm{M}$ para los biotipos pipón desgranador $y$ mechudo patechulo.

El proceso de germinación se vio favorecido para los dos biotipos por los tratamientos ethephon 6,92 $\times 10^{-}$ ${ }^{3} \mathrm{M}, \mathrm{AG}_{3}+\mathrm{FAP} 10^{-3} \mathrm{M}, \mathrm{AG}_{3}+\mathrm{FAP} 10^{-5} \mathrm{M}$, $\mathrm{KNO}_{3} 40 \mathrm{mM}, \mathrm{KNO}_{3} 30 \mathrm{mM}, \mathrm{KNO}_{3} 20$ $\mathrm{mM}, 20 / 30^{\circ} \mathrm{C}$, Luz amarilla y PEG $\left(30^{\circ} \mathrm{C}\right)$.

También se vio favorecido el proceso de germinación específicamente para pipón desgranador por $\mathrm{AG}_{3}+\mathrm{FAP} 10^{-3} \mathrm{M}$ 3h, Luz roja y luz roja/azul y para mechudo patechulo por $\mathrm{AG}_{3} 10^{-3} \mathrm{M}, \mathrm{AG}_{3} 10^{-5}, \mathrm{AG}_{3}+\mathrm{FAP} 10^{-}$ ${ }^{3} \mathrm{M} 5 \mathrm{~h}, 20^{\circ} \mathrm{C}$ y luz azul.

La ITG se vio favorecida para los dos biotipos por ethephon $6,92 \times 10^{-3} \mathrm{M}$, FAP $10^{-5} \mathrm{~m}, \mathrm{KNO}_{3} 40 \mathrm{Mm}, \mathrm{KNO}_{3}$ $30 \mathrm{mM}, \mathrm{KNO}_{3} 20 \mathrm{mM} .20^{\circ} \mathrm{C} 20 / 30^{\circ} \mathrm{C}$, Y PEG.

También se vio favorecido ITG específicamente para pipón desgranador por $\mathrm{AG}_{3}+\mathrm{FAP} 10^{-5} \mathrm{M}$, $\mathrm{AG}_{3}+\mathrm{FAP} 10^{-3} \mathrm{M} 5 \mathrm{~h}$, luz amarilla, Luz roja, luz azul y $40^{\circ} \mathrm{C}$, para mechudo patechulo por $\mathrm{AG}_{3} 10^{-3} \mathrm{M}, \mathrm{AG}_{3} 10^{-5} \mathrm{M}$, $\mathrm{AG}_{3}+\mathrm{FAP} 10^{-3} \mathrm{M}$ 3h y $\mathrm{AG}_{3}+\mathrm{FAP} 10^{-3} \mathrm{M}$ $5 \mathrm{~h}$. 
73

Los tratamientos ethephon $6,92 \times 10$ $3 \mathrm{M}$, KNO3 con 20,30 y $40 \mathrm{mM}$, la alternancia de temperaturas cardinales $\left(20^{\circ} / 30^{\circ} \mathrm{C}\right)$ y $30^{\circ} \mathrm{C}$ (PEG) promovieron mejor la germinación de los biotipos estudiados.

\section{RECOMENDACIÓN}

Los tratamientos en común para inducir la germinación y el ITG de los biotipos deben ser probados en bioensayos en otros grupos de arroz rojo como los rayones y varietales que también presentan dormancia.

\section{Referencias bibliográficas}

1. Carmona L. Conjuntura e diagnóstico do problema do arroz vermelho nos principais países produtores de arroz do mundo: Conjuntura e diagnóstico dos problemas do arroz vermelho na américa latina e Caribe. In: Anais do Seminário Latino Americano sobre Arroz Vermelho, editores Aldo Merotto Jr., Sérgio Iraçu Gindri Lopes, Augusto Kalsing, Porto Alegre, Brasil: 2013. p. 20-21.

2. Chauhan BS. Management strategies for weedy rice in Asia. Los Baños (Philippines): International Rice Research Institute; 2013.

3. Kaloumenos, NS, Capote N., Aguado A., Eleftherohorinos IG. Red rice (O. sativa) cross-resistance to imidazolinone herbicides used in resistant rice cultivars grown in northern Greece. Pest Biochem Physiol. 2013; 105(3): 177-183.

4. Ratnasekera D, Perera UIP, Zhuoxian H, Senanayake SGJN, Wijesekara GAW, Yang X, Baorong L. High level of variation among Sri Lankan Weedy rice populations, as estimated by morphological characterization. Weed Biol Manag. 2014; 14: 68-75.

5. Cho SY. Germination Characteristis of Korean and Southeast Asian Red rice (O. sativa L.) Seed as
Affected by temperature. Asian J Plant Sci. 2010; 9(2):104-107.

6. Xia HB, Xia H, Ellstry NC, Yang Ch, Lu BR. Rapid evolutionary divergence and ecotypic diversification of germination behavior in Weedy rice populations. New Phytol. 2011; 191:1119-1127.

7. Sale MA, Burgos NR, Shivrain VK, Murphy B, Gbur JrE. Morphological y Physiological Responses of Weedy Red Rice (O. sativa L.) and Cultivated Rice (O. sativa) to N Supply. Am J Plant Sci. $2011 ; 2: 569-577$.

8. Vargas JP. El arroz y su medio ambiente, Capitulo 6. En: Degiovanni B. Víctor, Martínez R. Cesar y Motta O. Francisco Editores. Producción Ecoeficiente del arroz en América Latina tomo I. Primera Edición, Publicación CIAT No. 370. Palmira Valle del Cauca; 2010. p. 83-97.

9. Xia HB, Xia H, Ellstry NC, Yang Ch, Lu BR. Rapid evolutionary divergence and ecotypic diversification of germination behavior in Weedy rice populations. New Phytol. 2011; 191:1119-1127.

10. Horn KJ, Nettles R, St. Clair SB. Germination response to temperature and moisture to predict distributions of the invasive grass red brome and wildfire. Biol invasions. 2015; DOI 10.1007/s10530-015-0841-3[online 2015-06-1].

11. Clavijo JF, Montealegre F. Arroz rojo en Colombia, comportamiento y riesgos, Capitulo 22. En: Degiovanni B. Víctor, Martínez R. Cesar y Motta O. Francisco Editores. Producción Ecoeficiente del arroz en América Latina tomo I. Primera Edición, Publicación CIAT No. 370. Palmira Valle del Cauca: 2010: p.413- 431. 
74

12. Merotto Jr A, Nunes $A L$, Markus C, Dalazen G, Bortol ED. Biotecnologia, transgênicos e novas tecnologias relacionadas com o controle Do arroz vermelho: Integração da biologia, biotecnologia e manejo para o controle de arroz vermelho. En: Aldo Merotto Jr., Sérgio Iraçu Gindri Lopes, Augusto Kalsing editores. Anais do Seminário Latino Americano sobre Arroz Vermelho. Porto Alegre, Brasil: 2013. p. 80-85.

13. International Seed Test Association ISTA. International rules for seed testing. Annexes Seed Sci Technol. 1993; 21(Supl): 288p.

14. Enríquez PEG, Suzán AH, Malda BG. Viabilidad y germinación de semillas de Taxodium mucronatum (Ten.). Agrociencia. 2004; 38: 375-381.

15. Bewley JD, Bradford KJ, Hilhorst HW, Nonogaki H. Seeds: Physiology of Development, Germination and Dormancy. New York, Heidelberg Dordrecht, London: Third edition. Springer; 2013.

16. Jendrzejczak $E$, Śmigerska K. Impact of presowing soaking of amaranth Seeds in solutions of growth regulators Part I. Effect of some growth regulators on Seed germination capacity. Acta Sci. Pol. 2014; 13(4): 4352.

17. Hilhorst HW. Part II Breaking Seed Dormancy and Styardizing Seed Dormancy Research Chapter 3. In: Seed Dormancy Methods and Protocols Edited by Allison R. Kermode New York Dordrecht Heidelberg London: Springer; 2011. p 43-52.

18. Huo H, Bradford KJ. Part I Seed Dormancy, Reviews, Research, and Perspectives Chapter 1 Molecular and Hormonal Regulation of Thermoinhibition of Seed Germination. In: Advances in Plant Dormancy. James
V. Yerson Editor. New York Dordrecht London: Springer Cham Heidelberg; 2015. p. 3-33.

19. Taiz L, Zeiger E. Plant Physiology. 5Th Ed. Sinauer Associates Inc., Publishers Sunderly, Massachusetts U.S.A; 2010.

20. Hu XW, Huang $X H$, Rong Wang YR. Hormonal and temperature regulation of seed dormancy and germination in Leymus chinensis. Plant Growth Regul. 2012; 67:199-207.

21. Wei $S$, Zhang Ch, Chen $X$, Li $X$, Sui $B$, Huang $H$, Cui $H$, Liu $Y$, Zhang $M$, Guo F. Rapid and Effective Methods for Breaking Seed Dormancy in Buffalobur (Solanum rostratum). Weed Sci. 2010; 58 (2): 141-146.

22. Noghani M, Shakori MJ, Darandeh N. Effect of cytokinin and gibberelin on lettuce seeds germination. Int J Biosci. 2014; 5(7):1-4.

23. Sebastian J, Wong MK, Tang E, Dinneny JR. Methods to Promote Germination of Dormant Setaria viridis Seeds. Plos One. 2014; 9(4) 1-7.

24. Rezvani M, Zaefarian F, Amini V. Effects of chemical treatments and environmental factors on seed dormancy and germination of shepherd's purse (Capsella bursa-pastoris (L.) Medic.). Acta Bot Bras. 2014; 28(4): 495-501.

25. Cavusoglu A, Sulusoglu $M$. The Effects of Exogenous Gibberellin on Seed Germination of the Fruit Species. DERLEME. 2015; 8(1): 6-9.

26. Finch SWE, Footitt S. Regulation of Seed Dormancy Cycling in Seasonal Field Environments Part I Chapter 2. In: Editor James V. Yerson Springer Cham Advances in Plant Dormancy. Heidelberg New York Dordrecht London: 2015: p 35-47. 
27. Prashant J, Norsworthy JK, Riley $M B$, Bridges $W$ y Jr. Annual Changes in Temperature and Light Requirements for Germination of Palmer Amaranth (Amaranthus palmeri) Seeds Retrieved from Soil. Weed Sci. 2010; 58 (4): 426432.

28. Moreno NEB, Miranda D, Martínez FEM. La zeatina fomenta la germinación de semillas de anón (Annona squamosa L.). Rev Colomb Cienc Hortic. 2013; 7(1) 9-19.

29. Roy A, Sahoo D, Tripathy BC. Light-hormone interaction in the redlight-induced suppression of photomorphogenesis in rice seedlings. Protoplasma. 2015; DOI 10.1007/s00709-015-0818-1[online 2015-05-31]

30. Fleishon S, Shani E, Ori N, Weiss D. Negative reciprocal interactions between gibberellin and cytokinin in tomato. New Phytol. 2011; 190 (3): 609-617.

31. Lei $B$, Ling $Y$, Jian AW, Hai SL. Effects of $\mathrm{KNO} 3$ pretreatment and temperature on seed germination of Sorbus pohuashanensis. J For Res. 2013; 24(2): 309-316.

32. Doria J. Generalidades sobre las semillas: su producción, conservación y almacenamiento. Cult Tr. 2010; 31(1): 74-85.

33. Tiansawat $P$, Dalling JW. Differential seed germination responses to the ratio of red to far-red light in temperate and tropical species. Plant Ecol. 2013; 214:751-764.

34. Zhang M, Yan Q, Zhu J. Optimum light transmittance for seed germination and early seedling recruitment of Pinus koraiensis: implications for natural regeneration. IFOREST. 2015; DOI: 10.3832/ifor1397-008[online 2015-0522].
35. Jacobsen JV, Barrero JM, Hughes T, Julkowska M, Taylor JM, Xu Q, Gubler $F$. Roles for blue light, jasmonate and nitric oxide in the regulation of dormancy and germination in wheat grain (Triticum aestivum L.). Planta. 2013; 238:121138. (76) 
Tabla 1. Factores y niveles aplicados a las semillas de los biotipos de arroz rojo.

\begin{tabular}{|c|c|}
\hline Factor & Nivel \\
\hline \multirow[t]{5}{*}{ Luz } & Luz azul (LA) \\
\hline & Luz roja (LR) \\
\hline & Luz amarilla (LAM) \\
\hline & Alterna luz roja 12 horas- \\
\hline & Luz azul 12 horas (Luz roja/azul) \\
\hline \multirow{3}{*}{$\begin{array}{l}\text { Temperatura } \\
\text { (temp) }\end{array}$} & $20^{\circ} \mathrm{C}$ \\
\hline & $\begin{array}{l}\text { 2. Termoperiodicidad } \\
\left(20 / 30^{\circ} \mathrm{C}\right)\end{array}$ \\
\hline & $40^{\circ} \mathrm{C}$ \\
\hline Ácido & $\mathrm{AG}_{3} 10^{-3} \mathrm{M}$ (Molar) \\
\hline \multicolumn{2}{|l|}{$\left(\mathrm{AG}_{3}\right)$} \\
\hline 6- furfiril & FAP $10^{-2} \mathrm{M}$ \\
\hline amino purina & FAP $10^{-3} \mathrm{M}$ \\
\hline$(\mathrm{FAP})$ & FAP $10^{-5} \mathrm{M}$ \\
\hline Ácido & $\mathrm{AG}_{3} 10^{-3} \mathrm{M}+\mathrm{FAP} 10^{-3} \mathrm{M}:$ \\
\hline giberélico+6- & $\left(\mathrm{AG}_{3}+\mathrm{FAP}\right) 10^{-3} \mathrm{M}$ \\
\hline furfuril amino & 2. $\quad \mathrm{AG}_{3} 10^{-5} \mathrm{M}+\mathrm{FAP} 10^{-}$ \\
\hline \multicolumn{2}{|l|}{$\left(\mathrm{AG}_{3}+\mathrm{FAP}\right)$} \\
\hline (aplicada en & $\mathrm{AG}_{3} 10^{-3} \mathrm{M}+\mathrm{FAP} 10^{-3} \mathrm{M}$ \\
\hline diferentes & 3horas: $\left(\mathrm{AG}_{3}+\mathrm{FAP}\right)$ 10-3 M 3h. \\
\hline & $\begin{array}{l}\text { 4. } \quad A_{3} 10^{-3} M+\text { FAP } 10^{-3} M \\
\text { 5horas: }\left(G_{3}+F A P\right) 10^{-3} M 5 h .\end{array}$ \\
\hline \multirow[t]{2}{*}{ Etileno (etil) } & Ethephon $1,4948 \times 10^{-2} \mathrm{M}$ \\
\hline & Ethephon 6,92 ×10-3M \\
\hline \multirow[t]{3}{*}{ Nitrato (nit) } & $\mathrm{KNO}_{3} 20 \mathrm{mM}$ (milimoles) \\
\hline & $\mathrm{KNO}_{3} 30 \mathrm{mM}$ \\
\hline & $\mathrm{KNO}_{3} 40 \mathrm{mM}$ \\
\hline Prueba & 1. $\quad 30^{\circ} \mathrm{C}, 12$ horas de luz y 12 \\
\hline $\begin{array}{l}\text { germinación } \\
\text { (PEG) }\end{array}$ & \\
\hline
\end{tabular}


Tabla 2. Análisis de varianza del diseño factorial teniendo como variable dependiente el porcentaje de germinación (\%G) y el ITG.

\begin{tabular}{|c|c|c|c|c|c|}
\hline \multicolumn{6}{|c|}{ Variable respuesta: Porcentaje de germinación (\%G) } \\
\hline Fuentes & G. L & S. C. & C. M. & $\begin{array}{l}\text { Valor } \\
\text { de F }\end{array}$ & $\begin{array}{l}\text { PR> } \\
F\end{array}$ \\
\hline Modelo & 43 & $\begin{array}{l}47280 \\
8\end{array}$ & 1099,6 & 5,1 & $* * *$ \\
\hline Error & 188 & $\begin{array}{l}40648 \\
3\end{array}$ & 216,2 & & \\
\hline Total & 231 & $\begin{array}{l}87929 \\
1\end{array}$ & & & \\
\hline $\mathrm{R}^{2}$ del modelo & 0,54 & & & & \\
\hline C.V. & 29,1 & & & & \\
\hline $\begin{array}{l}\text { Raíz cuadrada } \\
\text { del cuadrado } \\
\text { medio }\end{array}$ & 14,7 & & & & \\
\hline $\begin{array}{l}\text { Promedio } \\
\text { variable } \\
\text { respuesta }\end{array}$ & 50,6 & & & & \\
\hline \multicolumn{6}{|c|}{$\begin{array}{l}\text { Tipo de suma de cuadrados para realizar el análisis de la varianza: } \\
\text { tipo I }\end{array}$} \\
\hline F. V. & G. L & S. C. & C. M. & $\begin{array}{l}\text { Valor } \\
\text { de F }\end{array}$ & $P R>F$ \\
\hline Biotipo & 1 & 4005,6 & 4005,6 & $\begin{array}{l}18,5 \\
3\end{array}$ & *** \\
\hline Factor & 7 & $\begin{array}{l}16818 \\
2\end{array}$ & 2402,6 & $\begin{array}{l}11,1 \\
1\end{array}$ & *** \\
\hline Nivel & 7 & $\begin{array}{l}14720 \\
4\end{array}$ & 689,3 & 3,19 & *** \\
\hline BiotipoxFactor & 14 & 4824,9 & 1051,5 & 4,86 & $\star \star \star *$ \\
\hline $\begin{array}{l}\text { BiotipoxFactor } \\
\text { xNivel }\end{array}$ & 14 & 6911,8 & 493,7 & 2,28 & $* * *$ \\
\hline \multicolumn{6}{|c|}{ Variable respuesta: ITG } \\
\hline Fuentes & G. L & S. C. & C. M. & $\begin{array}{l}\text { Valor } \\
\text { de F }\end{array}$ & $\mathrm{PR}>\mathrm{F}$ \\
\hline Modelo & 43 & 0,122 & 0,0028 & 6,89 & $* * *$ \\
\hline Error & 188 & 0,078 & 0,0004 & & \\
\hline Total & 231 & 0,200 & & & \\
\hline $\mathrm{R}^{2}$ del modelo & 0,612 & & & & \\
\hline C.V. & 10,40 & & & & \\
\hline $\begin{array}{l}\text { Raíz cuadrada } \\
\text { del } \\
\text { cuadrado } \\
\text { medio }\end{array}$ & 0,020 & & & & \\
\hline $\begin{array}{l}\text { Promedio } \\
\text { variable } \\
\text { respuesta }\end{array}$ & 0,195 & & & & \\
\hline \multicolumn{6}{|c|}{$\begin{array}{l}\text { Tipo de suma de cuadrados para realizar el análisis de la varianza: } \\
\text { tipo I }\end{array}$} \\
\hline F. V. & G. L & S. C. & C. M. & $\begin{array}{l}\text { Valor } \\
\text { de F }\end{array}$ & $\mathrm{PR}>\mathrm{F}$ \\
\hline Biotipo & 1 & 0,011 & 0,011 & 26,08 & *** \\
\hline Factor & 7 & 0,046 & 0,007 & 16,00 & $\star * *$ \\
\hline Nivel & 7 & 0,041 & 0,003 & 7,16 & $\star * *$ \\
\hline BiotipoxFactor & 14 & 0,015 & 0,002 & 5,27 & $* * *$ \\
\hline $\begin{array}{l}\text { BiotipoxFactor } \\
\text { xNivel }\end{array}$ & 14 & 0,009 & 0,001 & 1,49 & ns \\
\hline
\end{tabular}

${ }^{* * *}: p \leq 0,001$; ns: no significativo; C. V.: Coeficiente de variación; F.V.: Fuentes de variación; G.L.: Grados de libertad; S.C.: Suma de cuadrados y C.M.: Cuadrado medio. 
Tabla 3. \% G e ITG de las semillas del biotipo significativamente diferentes con $\mathrm{P}<0,05$, usando la pipón desgranador (PD) y mechudo patechulo prueba de rango múltiple de Bonferroni. (MP) sometidas a diferentes niveles de los factores.

\begin{tabular}{|c|c|c|c|c|c|}
\hline $\begin{array}{c}\text { Niveles } \\
\text { Pipón } \\
\text { desgranado } \\
\text { r (PD) }\end{array}$ & \multicolumn{2}{|c|}{ (\%) G } & Niveles & \multicolumn{2}{|c|}{ ITG } \\
\hline $\begin{array}{c}\text { Ethephon } \\
6,92 \times 10^{-3} \\
M\end{array}$ & 91,00 & $a$ & $\begin{array}{c}\text { Ethephon } \\
6,92 \times 10^{-3} \\
M\end{array}$ & 0,2325 & $a$ \\
\hline LAM & 76,00 & $a b$ & PEG & 0,2306 & $a b$ \\
\hline $\begin{array}{c}\mathrm{AG}_{3}+\mathrm{FAP} \\
10^{-3} \mathrm{M} 3 \mathrm{~h}\end{array}$ & 74,00 & $a b$ & $\begin{array}{c}\mathrm{KNO}_{3} 30 \\
\mathrm{mM}\end{array}$ & 0,2200 & $a b$ \\
\hline $\begin{array}{c}\mathrm{AG}_{3}+\mathrm{FAP} \\
10^{-5} \mathrm{M}\end{array}$ & 68,50 & $a b$ & $20^{\circ} \mathrm{C}$ & 0,2150 & $a b c$ \\
\hline $\begin{array}{c}\mathrm{KNO}_{3} 40 \\
\mathrm{mM}\end{array}$ & 68,00 & $a b$ & $\begin{array}{c}\mathrm{AG}_{3}+\mathrm{FAP} \\
10^{-5} \mathrm{M}\end{array}$ & 0,2125 & $a b c$ \\
\hline luz roja/azul & 67,00 & $a b$ & FAP $10^{-5} \mathrm{M}$ & 0,2075 & $a b c d$ \\
\hline $\begin{array}{c}\mathrm{KNO}_{3} 30 \\
\mathrm{mM}\end{array}$ & 64,00 & $a b$ & $20 / 30^{\circ} \mathrm{C}$ & 0,2075 & $a b c d$ \\
\hline $\begin{array}{c}\mathrm{AG}_{3}+\mathrm{FAP} \\
10^{-3} \mathrm{M}\end{array}$ & 62,25 & $a b$ & $\begin{array}{c}\mathrm{KNO}_{3} 20 \\
\mathrm{mM}\end{array}$ & 0,2050 & $a b c d$ \\
\hline LR & 61,00 & $\begin{array}{c}a b \\
c\end{array}$ & LR & 0,2025 & $a b c d$ \\
\hline $20 / 30^{\circ} \mathrm{C}$ & 60,00 & $\begin{array}{c}\mathrm{ab} \\
\mathrm{c}\end{array}$ & $\begin{array}{c}\mathrm{KNO}_{3} 40 \\
\mathrm{mM}\end{array}$ & 0,2025 & $a b c d$ \\
\hline $\begin{array}{c}\mathrm{KNO}_{3} 20 \\
\mathrm{mM}\end{array}$ & 57,50 & $\begin{array}{c}\mathrm{ab} \\
\mathrm{c}\end{array}$ & $40^{\circ} \mathrm{C}$ & 0,2025 & $a b c d$ \\
\hline PEG & 57,06 & $\begin{array}{c}\mathrm{ab} \\
\mathrm{c}\end{array}$ & luz roja/azul & 0,1975 & abcde \\
\hline$\overline{L A}$ & 54,50 & $\mathrm{bc}$ & LAM & 0,1975 & abcde \\
\hline $40^{\circ} \mathrm{C}$ & 53,50 & $\mathrm{bc}$ & LA & 0,1900 & abcde \\
\hline $\mathrm{AG}_{3} 10^{-3} \mathrm{M}$ & 52,00 & $\mathrm{bc}$ & $\begin{array}{c}\mathrm{AG}_{3}+\mathrm{FAP} \\
10^{-3} \mathrm{M} 5 \mathrm{~h}\end{array}$ & 0,1900 & abcde \\
\hline $\mathrm{AG}_{3} 10^{-5} \mathrm{M}$ & 49,00 & $\begin{array}{c}\mathrm{bc} \\
\mathrm{d}\end{array}$ & $\begin{array}{c}\mathrm{AG}_{3}+\mathrm{FAP} \\
10^{-3} \mathrm{M}\end{array}$ & 0,1850 & bcde \\
\hline $20^{\circ} \mathrm{C}$ & 49,00 & $\begin{array}{c}\mathrm{bc} \\
\mathrm{d}\end{array}$ & $\begin{array}{c}\text { Ethephon } \\
1,4948 \times 10^{-} \\
{ }^{2} \mathrm{M}\end{array}$ & 0,1825 & bcde \\
\hline FAP $10^{-5} \mathrm{M}$ & 43,50 & $\begin{array}{c}\mathrm{bc} \\
\mathrm{d}\end{array}$ & $\mathrm{AG}_{3} 10^{-3} \mathrm{M}$ & 0,1800 & bcde \\
\hline FAP $10^{-3} \mathrm{M}$ & 41,00 & $\begin{array}{c}\mathrm{bc} \\
\mathrm{d}\end{array}$ & $\begin{array}{c}\mathrm{AG}_{3}+\mathrm{FAP} \\
10^{-3} \mathrm{M} 3 \mathrm{~h}\end{array}$ & 0,1775 & bcde \\
\hline $\begin{array}{c}\mathrm{AG}_{3}+\mathrm{FAP} \\
10^{-3} \mathrm{M} 5 \mathrm{~h}\end{array}$ & 26,00 & $\mathrm{~cd}$ & $\mathrm{AG}_{3} 10^{-5} \mathrm{M}$ & 0,1700 & cde \\
\hline $\begin{array}{c}\text { Ethephon } \\
1,4948 \times 10- \\
{ }^{2} \mathrm{M}\end{array}$ & 26,00 & $\mathrm{~cd}$ & FAP $10^{-3} \mathrm{M}$ & 0,1650 & de \\
\hline FAP $10^{-2} \mathrm{M}$ & 15,50 & $\mathrm{~d}$ & FAP $10^{-2} \mathrm{M}$ & 0,1550 & $\mathrm{e}$ \\
\hline
\end{tabular}

